\title{
DECONSTRUYENDO EL DISCURSO HEGEMÓNICO DEL CUERPO PERFECTO: EXPLORANDO LA FEMINIDAD A TRAVÉS DEL ANÁLISIS VISUAL DE FOLLETOS DE CLÍNICA DE ESTÉTICA
}

\author{
MARÍA MARTÍNEZ LIROLA \\ Universidad de Alicante \\ Research Fellow, Department of Linguistics, \\ University of South Africa (UNISA)
}

Recibido/12/03/2012

Aceptado/24/05/2012

\section{Resumen}

El cuerpo no es nunca una página en blanco sino que está lleno de sentido; éste puede considerarse un objeto transitorio impulsado por las ofertas del mercado. Las personas están atadas a sus cuerpos, de ahí que la cirugía estética sea una herramienta importante para las personas heterosexuales y transexuales pues nos permite modificarlo.

Este artículo explorará cómo se construye la feminidad a través del análisis visual de folletos de clínica de estética en los que aparecen representadas mujeres. El análisis explorará el tipo de mujeres que aparecen representadas, sus principales características y las relaciones entre ellas y los estereotipos tradicionales sobre la feminidad (mujeres atractivas, cuerpos esculturales, etc.).

Palabras clave: Gramática visual, texto, discurso, feminidad, clínicas de estética.

\section{Abstract}

The body is never a white page since it is always full of sense; it can be considered a transitory object, which is promoted by the offers of the market. People are tied to their bodies. For this reason, cosmetic surgery is an important tool for heterosexual and transsexual people because it allows to modify their bodies. 
This article will explore how femininity is created through the visual analysis of cosmetic clinics' leaflets in which women are represented. The analysis will explore the type of women that appear represented, their main characteristics, the relationships between them and the traditional stereotypes on femininity (attractive women, nice bodies, etc.).

Keywords: Visual grammar, text, discourse, feminity, cosmetic clinics. 


\section{Introducción}

Nos encontramos en un tiempo de Postfeminismo, de modo que los estudios sobre las mujeres han de buscar nuevos caminos y nuevas inquietudes con el fin de dar respuesta a las preguntas que las mujeres y los hombres se hacen en la actualidad y que van mucho más allá de las tradicionales cuestiones relacionadas con la igualdad y la diferencia. En la actualidad hemos de profundizar en cómo se construye la subyugación de las mujeres, cómo se construye la mujer, el lugar que ocupa su imagen en dicha construcción, hechos que están muy lejos de las afirmaciones de Witting ${ }^{1}$ sobre que no se nace mujer ni hay que llegar a serlo. Concurrimos con Burgos Díaz en que: «Las mujeres no constituyen un «grupo natural»; están socialmente integradas en un grupo de carácter artificial que la cultura establece para someter tanto las mentes como los cuerpos de las mujeres».

Los cuerpos de las mujeres son cosificados y normalmente oprimidos por el heterosexismo. Witting cuestiona la heterosexualidad y la dualidad de los sexos y los géneros, pues en esta concepción la mujer carece de libertad y forma parte de una sexualidad genitalizada y heterocentrada (Wittig, ${ }^{3}$; Wittig, ${ }^{4}$ ). Tal y como señala Burgos Díaz: «Sexo, género y sexualidad, son categorías culturales en las que se tejen nuestros cuerpos, nuestras emociones y pensamientos, nuestras vidas».

Este artículo cuestiona el papel que se le concede al cuerpo de la mujer en la heterosexualidad y señala el carácter normativo de la norma heterosexual, en la que las personas lesbianas, gays, bisexuales, transexuales e intersexuales son invisibilizadas. Es importante reivindicar la importancia de la mujer en su totalidad, sin centrarse tanto en el cuerpo como elemento

1. Wittig, Monique: «No se nace mujer», en Wittig, Monique: El pensamiento heterosexual y otros ensayos. Madrid, Editorial Egales, 2006, pp. 31-43.

2. BuRgos DíAz, Elvira. «Cruzando líneas. Trazando conexiones», op. cit., p. 38.

3. WitTig, Monique. Las guerrilleras. Barcelona, Seix Barral, 1971.

4. Wittig, Monique. El cuerpo lesbiano. Valencia, Pre-Textos, 1977.

5. Burgos DíAz, Elvira. Op.cit., p. 42. 
fundamental en su identidad, tal y como señala González Marín ${ }^{6}$ : «Las batallas del feminismo de todo tipo se han jugado y se juegan todavía en la arena de la oposición identidad/alteridad. O la mujer es otra y sin embargo no se comprende, o bien es otra como resultado de la construcción más o menos malevolente del patriarcado. ${ }^{7}$ »

Una de las ideas claves sobre la que se apoya este artículo es el concepto de 'biopoder' desarrollado por el sociólogo francés Foucault ${ }^{8}$. Este concepto se refiere a la práctica llevada a cabo por los Estados modernos de explotar numerosas y diversas técnicas para subyugar los cuerpos y controlar a la población. Foucault aplicó el análisis del discurso al estudio de la sociedad, en palabras de Mole": «Simply put, Foucault shows how discourses regulate what can be said, what can be thought and what is considered true or false, rational or irrational, legitimate or illegitimate, $[\ldots]$ ».

\section{Deconstruyendo el cuerpo: la imagen de la mujer en los medios de comunicación}

Las mujeres y los hombres no aparecen representadas/os de modo paritario en los medios de comunicación. Las mujeres aparecen como reclamo publicitario en el día de a día de los medios de comunicación (Abril Vargas ${ }^{10}$; Jeffries $^{11}$, Martínez Lirola ${ }^{12}$; Nuñez Puente y Establier Pérez ${ }^{13}$; Walter ${ }^{14}$ ). Cuando aparecen, lo que destaca es su aspecto físico, los mensajes se centran en los cuerpos de las mujeres, de modo que las cualidades intelectuales de las mismas suelen ser olvidadas, de modo que se dé respuesta a las demandas androcéntricas y

6. GonZÁlez Marín, Carmen. «Dos dogmas del feminismo». Feminismo/s 15 (2010), op. cit., p. 65.

7. La cursiva aparece en el texto original.

8. FouCAult, Michel. Historia de la sexualidad. Vol. I. La voluntad de saber. Madrid: Siglo XXI Editores, 1976.

9. Mole, Richard: «Discourse Identities/Identity Discourses and Political Power», en Mole, Richard. (ed.): Discursive Constructions of Identity in European Politics., op.cit., p. 16.

10. Abril VARGAS, Natividad: «Las mujeres de los media», en Hegoa (ed.): Utopía informativa. Propuestas para un periodismo más social. Bilbao: Hegoa, 2007, pp. 24-28.

11. JefFries, Lesley. Textual Construction of the Female Body. A Critical Discourse Approach. Palgrave, Macmillan, 2007.

12. Martínez Lirola, María. «Explorando la invisibilidad de mujeres de diferentes culturas en la sociedad y en los medios de comunicación». Palabra Clave 13, 1 (2010a), pp. 161-173.

13. NuÑEZ PuENTE, Sonia y Helena ESTABLIER PÉREZ (eds.) La representación/presencia de la mujer en los medios de comunicación. Feminismos, 11, 2008.

14. WALter, Natasha. Muñecas vivientes. El regreso del sexismo. Madrid: Turner Publicaciones, 2010. 
patriarcales de la sociedad (Irigaray ${ }^{15}$; Conboy ${ }^{16}$; López Díez ${ }^{17}$; Martínez Liro$\left.1 a^{18} ; N_{\text {Úñez }}{ }^{19}\right)$.

De la afirmación presentada en el párrafo anterior se deduce que los cuerpos de las mujeres no aparecen representados del mismo modo que los de los hombres y por tanto no podemos hablar de igualdad entre mujeres y hombres, tal y como ocurre en muchos aspectos de la vida social. Por esta razón, la Ley Orgánica 3/2007 $7^{20}$, de 22 de marzo, promovida por el Gobierno central de España en manos del Partido Socialista, surge con la finalidad de lograr una igualdad efectiva de mujeres y hombres. De ahí que el título 3 de la citada Ley dedicado a 'Igualdad y medios de comunicación', y más concretamente el artículo 36, dedicado a 'La igualdad en los medios de comunicación social de titularidad pública', ponga de manifiesto la necesidad de velar por una representación igualitaria de mujeres y hombres: «Los medios de comunicación social de titularidad pública velarán por la transmisión de una imagen igualitaria, plural y no estereotipada de mujeres y hombres en la sociedad y promoverán el conocimiento y la difusión del principio de igualdad entre mujeres y hombres».

Los medios de comunicación tienen un papel fundamental en la transmisión de realidades, en la construcción de ideología y en la educación. En general, los medios emplean textos en los que predominan las imágenes con el fin de ilustrar de modo gráfico los significados que quieren transmitir. Esto hace que sean muchos los autores que hablen del carácter multimodal de las sociedades actuales, es decir, se trata de sociedades en las que hay más de un modo de comunicación, es decir, la comunicación es escrita, visual, musical, etc. (Baldry y Thibault ${ }^{21}$; Kress y van Leeuwen ${ }^{22}$;

15. IRIgARAY, Luce. Ese sexo que no es uno. Madrid, Saltés, 1982.

16. Conboy, Martin. Language of the News. Londres, Routledge, 2007.

17. LÓPEZ DíEZ, Pilar. «Los medios y la representación de género: algunas propuestas para avanzar». Feminismos 11 (2008), pp. 95-108.

18. MARTínez LiROlA, María «Explorando nuevas formas de violencia de género: la mujer como objeto en los folletos de clínicas de estética». Global Media Journal Mexico, 7, 13, (2010b), pp. 80-94.

19. NúNEZ PUENTE, Sonia: «En brazos de la mujer fetiche: Nacimiento y pervivencia de la imagen de la mujer como fetiche», en Santaemilia, José. (ed.): Género, lenguaje y traducción. Actas del Primer Seminario Internacional sobre Género y Lenguaje. Valencia: Universidad de Valencia, 2003, pp. 108-134.

20. LEY ORGÁNICA 3/2007, de 22 de marzo, para la igualdad efectiva de mujeres y hombres. Páginas 12611-12645. BOE 71 de 23/03/2007. Referencia 2007/06115.

21. BALDRY, Anthony y ThiBAult, Paul J. Multimodal Transcription and Text Analysis. Londres, Equinox, 2006.

22. KRESS, Gunther y VAN LeEUWEN, Teun. «Colour as a semiotic mode: Notes for a grammar of colour». Visual Communication, 1, 3, (2002), pp. 343-368. 
Kress $^{23}$; Macken-Horarik ${ }^{24}$ ). En la siguiente sección se presentará un análisis de dos folletos de clínica de estética en los que la imagen de la mujer juega un papel fundamental en el modo en que el texto construye su significado.

\section{Análisis de la imagen de la mujer en los folletos de clínicas de estética}

La cirugía estética hace posible que los cuerpos que no se adaptan a la norma puedan ser modificados para adaptarse al 'deber ser' establecido socialmente, siempre con el riesgo de poder perder la vida en el quirófano, tal y como ocurre en todas las operaciones. El 'deber ser' lo establecen los grupos de poder, es decir, el sistema patriarcal que enmarca nuestro proceso de socialización desde que nacemos. Las clínicas de cirugía estética no tratan igual los cuerpos de las mujeres y los de los hombres en su publicidad, de modo que se pone de manifiesto que las mujeres se representan como un sexo inferior, tal y como señala Despentes ${ }^{25}$ :

Nunca iguales, nuestros cuerpos de mujer. Nunca seguras, nunca como ellos. Somos el sexo del miedo, de la humillación, el sexo extranjero. Su virilidad, su famosa solidaridad masculina, se construye a partir de esta exclusión de nuestros cuerpos, se teje en esos momentos. Es un pacto que reposa sobre nuestra inferioridad. Sus risas de tíos, entre ellos, la risa de los más fuertes, de los más numerosos.

Para llevar a cabo este estudio recopilamos los folletos repartidos por la clínica de estética Dorsia durante los años 2007-2009. Se optó por esta clínica de estética porque su publicidad era muy agresiva en estos años y por ser la clínica número uno en intervenciones de cirugía estética en España. Obtuvimos un total de 20 folletos. En todos los folletos recopilados excepto en uno, encontramos a una mujer joven y blanca como protagonista. Ésta suele aparecer sola en posición seductora o acompañada por algún hombre, en el que su posición y su gesto indica que posee a la mujer.

23. KreSS, Gunther. Multimodality. A Social Semiotic Approach to Contemporary Communication. London: Routledge, 2010.

24. MACKEN-HORARIK, Mary. «Interacting with the multimodal text: reflections on image and verbiage in ArtExpress». Visual Communication, 3, 1, (2004), pp. 5-26.

25. DesPentes, Virginie. La teoría King Kong. Op.cit., p. 30. 

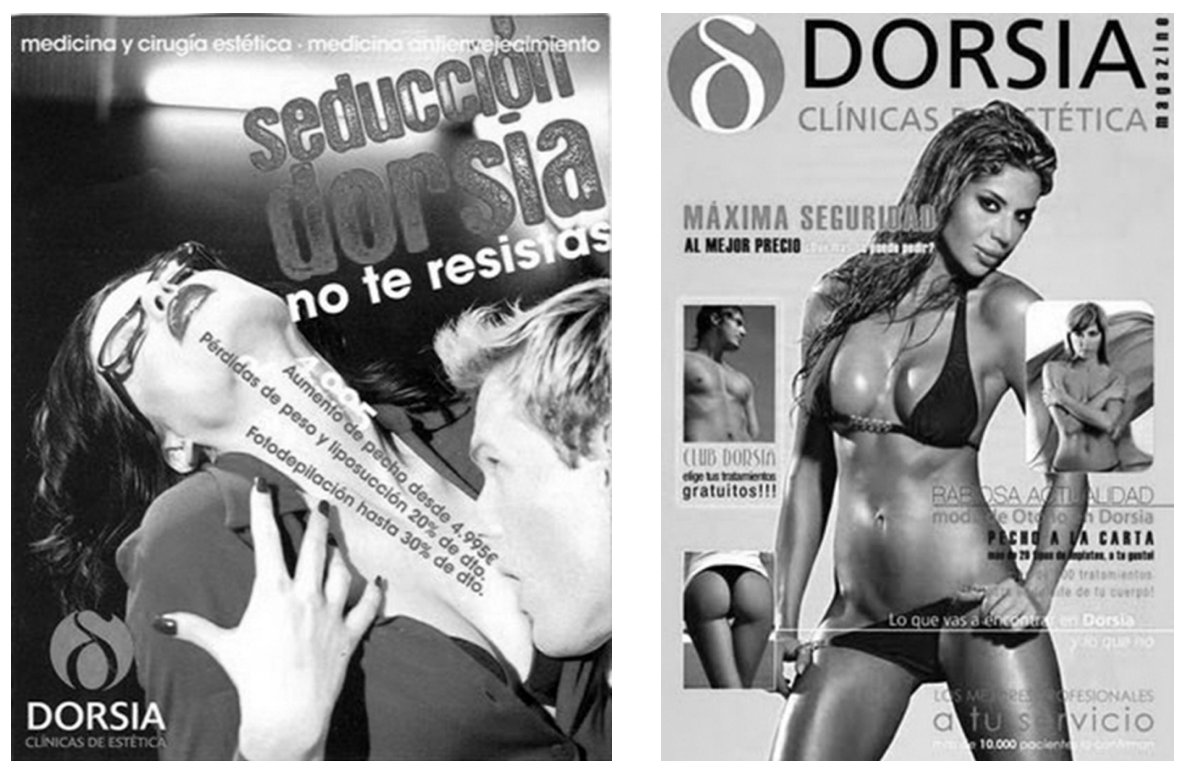

En los párrafos siguientes de este artículo ofrecemos un análisis de las principales características lingüísticas y visuales en dos de los 20 textos recopilados. Se han elegido estos dos por ser representativos del modo en que la mujer aparece representada en este tipo de textos: con poca ropa o con un gran escote de modo que se la represente casi desnuda o se muestre gran parte de su cuerpo, en palabras de Preciado ${ }^{26}$ : «El desnudo público como categoría social y política, como trasgresión legal o moral, pero también como espectáculo, es una invención reciente. Sólo la modernidad ha utilizado el desnudo femenino hasta transformarlo en una práctica al mismo tiempo codificada y mercantilizable».

El análisis que se ofrece nos permite deconstruir los textos y entender la lengua escrita y las características visuales de los textos que son objeto de estudio como forma de comportamiento social y de práctica social, tal y como señala Blackledge ${ }^{27}$ : «Social life can be seen as networks of diverse social practices, including economic, political, cultural, familiar practices and so on. Social practices are more-or-less stable forms of social activity which always, or almost always, include discourse».

26. Preciado, Beatriz. Pornotopía. Arquitectura y sexualidad en «Playboy» durante la guerra fría. Op. cit., p. 75.

27. BlaCKLEDGE, Adrian. Discourse and Power in a Multilingual World. Op. cit., p. 4. 
En los siguientes párrafos vamos a describir las principales características visuales y lingüísticas de los textos que son objeto de análisis con la finalidad de deconstruir y comprender el significado que hay oculto y explícito en los textos multimodales producidos por Dorsia. Dichos folletos se producen con el fin de persuadir a las mujeres a que acudan a la cirugía estética con el fin de modificar sus cuerpos y adaptarlos a los cánones de la sociedad.

En este sentido, pretendemos profundizar en la idea del cuerpo como texto, como discurso 'territorializable' que está sujeto a los vaivenes de la estética a los patrones culturales y mercado laboral. De ahí que sea importante tener presente que cada cuerpo es distinto, de igual modo que es distinta cada experiencia de la feminidad y de la masculinidad, en palabras de Ziga ${ }^{28}$ : «No hay dos experiencias con la feminidad ni con la masculinidad idénticas, el contexto y la percepción de los propios devenires son también aquí únicos».

Con respecto a las características visuales (Kress y van Leeuwen ${ }^{29}$ ), una de las más importantes de la composición de estos textos en los que se combina la imagen con el texto escrito es la prominencia que ha de entenderse como el resultado de la interacción de una serie de factores (colores, lugar en que aparece la imagen, tamaño de la imagen, tamaño de las letras, etc.) y en especial de observar el tamaño de la imagen con relación al texto escrito. Es evidente que en los dos textos que son objeto de estudio la imagen de la mujer es el elemento predominante pues aunque la cirugía estética también se ofrece a los hombres, son las mujeres el público prioritario, tal y como señala Preciado ${ }^{30}$ :

Existe una institucionalización desigual del cuerpo masculino y femenino en los circuitos de producción de saber y de artefactos biomédicos. El cuerpo de las mujeres, incluso de aquellas que aparecen como normales, las femeninas, las heterosexuales, las que no son ni frígidas ni histéricas, ni putas ni ninfómanas, el cuerpo de las perfectas madres potenciales, está de todos modos siempre sujeto a vigilancia y a regulación. Por definición, el cuerpo femenino nunca es completamente normal fuera de las técnicas que hacen de él un cuerpo social.

Además, la composición de los textos multimodales también presta atención a los marcos pues éstos indican si los elementos que configuran el texto están o no unidos. En el texto 1 observamos que el marco es no marcado y en el texto 2 hemos de destacar que las imágenes de personas que aparecen rodeando a

28. ZIGA, Itziar. Devenir Perra. Op. cit., p. 40.

29. KRESS, Gunther y VAN LeEUWEN, Teun. Reading Images: The Grammar of Visual Design. Segunda edición. Londres, Routledge Press, 2006.

30. PreCIAdo, Beatriz. Testo Yonqui. Op. cit., p. 147. 
la protagonista aparecen enmarcadas y en tamaño mucho menor que el de la mujer protagonista que aparece en el centro del texto.

Finalmente, una de las características fundamentales de la composición de los textos multimodales es el valor de la información que aparece a la derecha y a la izquierda de cada texto. Normalmente a la derecha encontramos la información nueva y a la izquierda encontramos la información conocida. En el texto 1 la información nueva se encuentra a la derecha de modo que se le concede importancia al hombre que aparece en el texto; en el texto 2, la información nueva aparece en la mujer que se encuentra en el centro del texto.

Al referirnos al valor de la información, también tiene importancia lo que se considera ideal y real, es decir, lo que aparece en la parte superior e inferior de la página. La parte superior de la página tiene más importancia y suele coincidir con lo ideal, de ahí que en los dos textos analizados encontremos el fin de Dorsia: «Medicina y cirugía estética-Medicina antienvejecimiento» (texto 1) y «Dorsia, Clínicas de Estética» (texto 2). El hecho de que esta información aparezca en la parte superior de los textos que son objeto de estudio tiene como objetivo persuadir a las lectoras para que perciban la cirugía estética como un tipo de medicina que potencia la salud. Por otro lado, la parte inferior de la página suele contener la información real, de ahí que sea ahí donde aparece la mayor parte del cuerpo de las protagonistas en los textos analizados, aunque en ambos casos, la cabeza de la mujer se sitúa en la parte superior de la página.

Finalmente, el valor de la información también presta atención a la parte del mensaje que encontramos en el centro y en los márgenes. El centro suele coincidir con el núcleo de la información, de ahí que en el texto 1 , en el centro encontramos las ofertas que Dorsia ofrece que, tal y como puede observarse, aparecen en línea recta con el pecho de la mujer y con los labios. Además, hemos de destacar que en el texto 2 , justo en el centro de la imagen principal encontramos los pechos de la mujer ligeramente cubiertos. En los márgenes encontramos la información que tiene menos importancia, de ahí que en ambos textos aparezca el número de registro sanitario de Dorsia en el margen izquierdo, al tratarse de una información poco relevante para las lectoras y difícil de recordar.

El color es un elemento importante en el análisis visual. En los textos analizados los colores elegidos para la ropa de la mujer son el rojo (vid. texto 1) y el verde (vid. texto 2 ). Heller ${ }^{31}$ señala que el rojo es el color de las pasio-

31. Heller, Eva. Psicología del color. Cómo actúan los colores sobre los sentimientos y la razón. Barcelona, Gustavo Gili, 2004. 
nes, del amor al odio, del peligro, etc. En el texto 1, el color rojo representa la pasión y encaja perfectamente con la postura de la mujer, que es claramente pasional. Por otro lado, en el texto 2, el traje de baño de la mujer es de color verde. Heller, pone de manifiesto que el verde es el color de la fertilidad, de la esperanza y de la burguesía y lo considera un color intermedio, aunque la mujer que luce el traje de baño está en una postura provocativa. Concurrimos con Kress y van Leeuwen ${ }^{32}$ en que los colores que configuran los textos multimodales, incluida la ropa, son empleados para destacar algunas características de las personas representadas, de ahí que en el caso de los textos que son objeto de estudio podamos afirmar que las mujeres que aparecen en primer plano son mujeres atractivas que muestran parte del cuerpo del que están orgullosas.

Otra característica visual importante es el lugar en el que aparece el logotipo de Dorsia en ambos textos: aparece al final de la página y a la izquierda en el texto 1 y por el contrario, al comienzo de la página pero también a la izquierda en el texto 2. En este último caso, el logotipo se destaca más porque aparece en la parte superior del folleto, que como hemos dicho en párrafos anteriores tiene más importancia.

Además, otro contraste entre ambas imágenes es que en la primera el fondo es oscuro y por lo tanto contrasta con el color de la piel de los protagonistas y de la ropa. En el texto 2 el fondo es claro, y aunque se diferencia perfectamente el color de la piel de la protagonista y se puede leer el texto escrito no se destaca tanto como en el primer texto.

Por otro lado, la mujer representada en el primer texto es morena y contrasta con la mujer del texto 2 que es más rubia pues tiene mechas en el pelo (la mujer que aparece enmarcada a la derecha de la protagonista es rubia). Sin embargo, el hombre en el texto 1 es rubio y el hombre que aparece enmarcado en el lado izquierdo de la protagonista en el texto 2 es moreno.

Con respecto al tamaño de la imagen de la mujer protagonista de ambos textos, en los dos casos es bastante grande pues ocupa más de la mitad del folleto y aparece en primer plano. Esto contrasta con los hombres que aparecen representados en ambos textos pues en el caso del texto 1 su tamaño es pequeño, pues sólo aparece su cabeza pero también aparece en primer plano. Sin embargo el hombre que aparece en el texto 2, además de ocupar un pequeño espacio en el texto, aparece a la izquierda, lugar que, como hemos dicho, tiene menos importancia en la composición de los textos multimodales.

32. KRESS, Gunther y VAN LEEUWEN, Teun. «Colour as a semiotic mode: Notes for a grammar of colour». Visual Communication, 1, 3, op. cit., p. 347. 
Al referirnos a la posición del cuerpo de la protagonista, hemos de destacar que en los dos casos aparece en movimiento, de modo que el folleto tiene viveza y dinamismo. En el caso del texto 1, la mujer aparece inclinada hacia atrás, en manos del hombre. Además, esta mujer aparece representada con gafas, con la boca entreabierta, su piel es muy blanca y tiene un buen cuerpo. La expresión facial de esta mujer nos indica que está disfrutando, que siente placer. Ella no mira a las lectoras. La mujer interactúa con el hombre en la imagen pues él le está besando el pecho y esto le provoca placer. Sólo él la mira a ella. En el caso del texto 2, la mujer muestra su cuerpo casi por completo. Su piel es muy morena y al igual que en el ejemplo anterior, también tiene un buen cuerpo. Esta mujer sí mira a las lectoras directamente, su mirada es desafiante y el hecho de que mire directamente pone de manifiesto que requiere una respuesta directa de las lectoras. En ambos casos se invita a las mujeres a tener un cuerpo como el de ellas.

También es un elemento importante en la composición de los textos multimodales el tamaño de las letras que aparecen en los mismos. En los dos textos analizados el titular de ambos aparece con un tamaño de letra mayor y en negrita en ambos textos. Esta característica puede entenderse a medio camino entre las características visuales y verbales de los textos multimodales.

Aunque en los dos textos la imagen es el elemento más destacado, también es necesario referirnos a las características verbales que encontramos en los dos textos. El titular del texto 1 es «Seducción Dorsia. No te resistas». Encontramos una colocación que establece que hay un tipo de seducción denominada Dorsia. Además tenemos un imperativo en negativo utilizado con el fin de persuadir a las/los lectores. En el texto 2, además de aparecer «Dorsia, Clínicas de estética» en la parte superior del texto, a la altura del cuello de la protagonista encontramos en negrita: «Máxima seguridad al mejor precio. ¿Qué más se puede pedir?» En esta ocasión, además de los adjetivos máxima y mejor con el fin de destacar, el recurso lingüístico empleado es una pregunta retórica.

Los recursos lingüísticos más empleados por los publicistas de Dorsia son las expresiones enfáticas, las exclamaciones y las repeticiones. Las principales colocaciones y expresiones enfáticas con el fin de indicar ponderación o sobreénfasis que aparecen en ambos textos son: «Medicina antienvejecimiento» (texto 1); «rabiosa actualidad», «club Dorsia» y «pecho a la carta» (texto 2). En el caso del texto 2, se emplean varias exclamaciones con el fin de destacar: en los dos primeros ejemplos sólo aparece la exclamación final (gratuitos!!!, a tu gusto!), y en el último ejemplo encontramos los signos de exclamación al principio y al final de la misma (¡y más de 500 tratamientos para el deleite 
de tu cuerpo!). Además, en este texto se repite el adjetivo mejor: «mejor precio», «mejores profesionales» y se destacan los números: «más de 20 tipos de implantes», «más de 500 tratamientos», «más de 10000 pacientes». En el texto 1 , encontramos ausencia de verbos en los tratamientos que ofrecen. Sólo aparece un verbo en imperativo: «No te resistas». En el texto 2 encontramos varias formas verbales: un imperativo (elige tus tratamientos gratuitos!!!), dos infinitivos (pedir y encontrar) y un presente (confirman).

El análisis lingüístico llevado a cabo nos permite observar que en ningún momento se feminiza la lengua escrita a pesar de que las protagonistas de los textos analizados son mujeres y son folletos dirigidos fundamentalmente a mujeres. En este sentido, nos parece que las mujeres son usadas como protagonistas en estos folletos con el fin de persuadir a otras mujeres para que tengan un cuerpo similar y concedan importancia a su imagen, de modo que cumplan con los cánones establecidos y respondan a las demandas sociales.

\section{A modo de conclusión}

Uno de los fines del cuerpo de la mujer ha sido siempre el de agradar al hombre, el de responder a los cánones de belleza establecidos por el patriarcado con el fin de ser bonita y aceptada por la sociedad. De ahí que en los dos folletos de la clínica de estética Dorsia analizados encontramos mujeres jóvenes, esbeltas y delgadas. En ambos folletos la mujer presenta una postura provocativa que hace que sea percibida como un objeto sexual.

El análisis de cómo se articulan las los significados lingüísticos y visuales en los textos analizados llevado a cabo en el apartado anterior nos permite hablar de la cosificación del cuerpo de la mujer: el cuerpo está utilizado como reclamo publicitario, es un cuerpo adorado como portador de fertilidad, construido según los deseos masculinos, ideas fundamentales en el contexto social y cultural marcado por el patriarcado y el androcentrismo en que estos textos se enmarcan. De este modo se observa que la capacidad racional de las mujeres queda en segundo plano pues lo que se destaca con los textos analizados es su rol estético al ser representada como un objeto decorativo, para adornar, es decir, en este caso son empleadas para promocionar a la clínica de cirugía estética Dorsia.

Los textos analizados contribuyen a la aportación de la construcción de la feminidad y la identidad sexual, fundamentalmente heterosexual, siendo las clínicas de estética elementos de biopoder que definen el binarismo de género y presentan una imagen particular de las mujeres: responder a los cánones de belleza establecidos por la sociedad de ahí que tengan que ser 
guapas, delgadas y con un cuerpo que se caracterice por ser estilizado y bien proporcionado.

Se trata, pues, de textos que potencian el culto al cuerpo y la necesidad de mantener el cuerpo joven por medio de la cirugía estética, de ahí que se hable de «medicina antienvejecimiento» en el texto l. Es decir, se presentan mujeres con cuerpos perfectos que agradan a los hombres, de modo que lo masculino impera sobre lo femenino, característica fundamental del sistema patriarcal que envuelve nuestra sociedad.

El deseo por adaptarse a los cánones y cumplir así con las demandas sociales lleva a algunas mujeres a hacerse operaciones de cirugía estética a veces muy poco seguras con el fin de cambiar sus cuerpos de modo que su imagen física mejore teóricamente.

\section{Referencias bibliográficas}

ABRIL VARGAS, Natividad: «Las mujeres de los media», en Hegoa (ed.): Utopía informativa. Propuestas para un periodismo más social. Bilbao: Hegoa, 2007, pp. 24-28.

BALDRY, Anthony y THibAult, Paul J. Multimodal Transcription and Text Analysis. Londres, Equinox, 2006.

Blackledge, Adrian. Discourse and Power in a Multilingual World. Amsterdam/ Philadelphia, John Benjamins, 2009.

Burgos DíAZ, Elvira. «Cruzando líneas. Trazando conexiones». Feminismo/s 15 (2010), pp. 33-54.

Conboy, Martin. Language of the News. Londres, Routledge, 2007.

DESPENTES, Virginie. La teoría King Kong. Barcelona, UHF, 2009.

FouCAULT, Michel. Historia de la sexualidad. Vol. I. La voluntad de saber. Madrid: Siglo XXI Editores, 1976.

GonZÁlez MARín, Carmen. «Dos dogmas del feminismo». Feminismo/s 15 (2010), pp. 55-74.

Heller, Eva. Psicología del color. Cómo actúan los colores sobre los sentimientos y la razón. Barcelona, Gustavo Gili, 2004.

IrIGARAY, Luce. Ese sexo que no es uno. Madrid, Saltés, 1982.

JefFries, Lesley. Textual Construction of the Female Body. A Critical Discourse Approach. Palgrave, Macmillan, 2007.

KRESS, Gunther. Multimodality. A Social Semiotic Approach to Contemporary Communication. London: Routledge, 2010.

KRESS, Gunther y VAN LeEUWEN, Teun. "Colour as a semiotic mode: Notes for a grammar of colour». Visual Communication, 1, 3, (2002), pp. 343-368.

KresS, Gunther y VAN LeEUwEN, Teun. Reading Images: The Grammar of Visual Design. Segunda edición. Londres, Routledge Press, 2006. 
LEY ORGÁNICA 3/2007, de 22 de marzo, para la igualdad efectiva de mujeres y hombres. Páginas 12611-12645. BOE 71 de 23/03/2007. Referencia 2007/06115

LÓPEZ DíEZ, Pilar. «Los medios y la representación de género: algunas propuestas para avanzar». Feminismos 11 (2008), pp. 95-108.

MACKEN-HORARIK, Mary. «Interacting with the multimodal text: reflections on image and verbiage in ArtExpress». Visual Communication, 3, 1, (2004), pp. $5-26$.

MARTínez Lirola, María. «Explorando la invisibilidad de mujeres de diferentes culturas en la sociedad y en los medios de comunicación». Palabra Clave 13, 1 (2010a), pp. 161-173.

Martínez LiRola, María. «Explorando nuevas formas de violencia de género: la mujer como objeto en los folletos de clínicas de estética». Global Media Journal Mexico, 7, 13, (2010b), pp. 80-94. http://www.gmjei.com/journal/index. php/GMJ_EI/article/viewArticle/292, consultado el 27-01-2011.

Mole, Richard: «Discourse Identities/Identity Discourses and Political Power», en Mole, Richard. (ed.): Discursive Constructions of Identity in European Politics. Londres: Palgrave Macmillan, 2007, pp. 1-21.

NúÑEZ PUENTE, Sonia: «En brazos de la mujer fetiche: Nacimiento y pervivencia de la imagen de la mujer como fetiche», en Santaemilia, José. (ed.): Género, lenguaje y traducción. Actas del Primer Seminario Internacional sobre Género y Lenguaje. Valencia: Universidad de Valencia, 2003, pp. 108-134.

Nuñez Puente, Sonia y Helena ESTABlier PÉREz (eds.) La representación/presencia de la mujer en los medios de comunicación. Feminismos, 11, 2008.

Preciado, Beatriz. Testo Yonqui. Madrid: Espasa Calpe, 2008.

Preciado, Beatriz. Pornotopía. Arquitectura y sexualidad en «Playboy» durante la guerra fría. Barcelona: Anagrama, 2010.

WALter, Natasha. Muñecas vivientes. El regreso del sexismo. Madrid: Turner Publicaciones, 2010.

WitTig, Monique. Las guerrilleras. Barcelona, Seix Barral, 1971.

Wittig, Monique. El cuerpo lesbiano. Valencia, Pre-Textos, 1977.

WitTiG, Monique: «No se nace mujer», en Wittig, Monique: El pensamiento heterosexual y otros ensayos. Madrid, Editorial Egales, 2006, pp. 31-43.

ZIGA, Itziar. Devenir Perra. Madrid, Melusina, 2009. 